\title{
Overload Control Strategy Based on Triangular Fuzzy Analytic Hierarchy Process
}

\author{
Heng Wang ${ }^{1}$ \\ ${ }^{1}$ School of Electrical Engineering, North China Electric Power University, Changping, Beijing, China
}

\begin{abstract}
After the high-voltage transmission line is cut off due to a fault, the bearing transmission power will be transferred, which may cause overload. If overload cannot be eliminated quickly and scientifically, it is very likely that the line backup protection will be activated, which will trigger chain trip or even blackout accidents. In view of this, this paper proposed a cross-voltage level optimization load shedding control strategy based on fuzzy analytic hierarchy process. Through step-by-step progression in the three indexes of line overload degree, load importance degree, and unit load shedding cost, the lower-level lines of the overload line are selected for optimal line removal, thus achieving the goal of optimizing load shedding across voltage levels.
\end{abstract}

\section{1 introduction}

In recent years, most large-scale power outages at home and abroad are caused by fault and other factors. Some important high-voltage transmission lines are cut, resulting in transfer of the bearing transmission power and circuitous transmission through other transmission lines with longer electrical distances. If the power of these lines exceeds their corresponding rated power, overload will occur. At this time, the backup protection is very likely to operate unreasonably, which will cause chain trip events and even lead to blackouts, inflicting incalculable loss to the society and economy. The $500 \mathrm{kV}$ grid power flow transfer overload will trigger protection action, so it is necessary to quickly eliminate the overload. However, most of the existing load shedding strategies fall within the category of secondary and third defense lines with start time lagging. When the load shedding function is implemented, the system may induce protection action due to overload, but it is unreasonable to blindly cut 500 $\mathrm{kV}$ high voltage overload line, as it not only enlarges the blackout range and affects power supply of some important loads, but also may fail to reduce the load, just accelerating the chain trip instead, thus causing large-scale blackout. Therefore, we need to proceed from the overall system, select the appropriate line load for shedding in the downstream of the overload line, that is, in the low-voltage level network, which can effectively solve the overload problem of the upstream line and also ensure the normal power supply of important loads to minimize the impact on the system and users.

At present, the main load shedding schemes include linear programming algorithm ${ }^{[1]}$, particle swarm algorithm ${ }^{[2]}$, adjustment of equal and opposite quantities in pairs ${ }^{[3]}$, interlocking load shedding scheme based on the transmission section $\mathrm{N}-1$ static secure power flow constraint ${ }^{[4]}$. The main principle of Analytic Hierarchy Process (AHP) is to simplify the entire complex problem into a multi-level structure of target level, criterion level, and program level from top to bottom. Then, the levels are ranked according to certain principles to determine the weight of each level compared with the upper layer. Its rationality is proved through consistency test. Finally, the weight of each factor for the overall decision-making goal is ranked. Load shedding optimization is to find the most reasonable scheme through gradual screening, which basically conforms to the principle of analytic hierarchy process. However, the uncertainty and ambiguity in the experts' understanding of the evaluation factors can easily reduce the credibility in calculation-based vector ordering. Therefore, to reduce deviations, this paper introduced fuzzy numbers, and combined the traditional analytic hierarchy process with triangular fuzzy mathematics (TFN) to form TFN-AHP triangular fuzzy analytic hierarchy process ${ }^{[5]}$, which replaced point value with fuzzy number to form a judgment matrix. Then, it calculated the fuzzy number comprehensive weights and sort them.

email: 1182201004@ncepu.edu.cn 


\section{Triangular fuzzy analytic hierarchy process}

\subsection{Basic concepts of triangular fuzzy mathematics}

Denote $F(R)$ as all the fuzzy numbers on $R$, let $M \in F(R)$, if:

(1) The membership function of $\mathrm{M} \mu_{m}: \mathrm{R} \rightarrow[0,1]$ can be expressed as:

$$
\mu_{m}(x)=\left\{\begin{array}{cl}
\frac{x}{m-l}-\frac{l}{m-l} & x \in[\mathrm{l}, \mathrm{m}] \\
\frac{x}{m-u}-\frac{u}{m-u} & x \in[\mathrm{m}, \mathrm{u}] \\
0 & \text { other }
\end{array}\right.
$$

Where, $1 \leq \mathrm{m} \leq \mathrm{u}$;

(2) There is $x_{0} \in R$, so that $\mu_{m}\left(x_{0}\right)=1$;

(3) For any $\lambda \in(0,1), M=\left\{x \mid \mu_{m}(x) \geq \lambda\right\}$ is a convex set;

Then, $M$ is called triangular fuzzy number, which can be recorded as $(1, \mathrm{~m}, \mathrm{u})$.
(3) Calculate and adjust the judgment matrix

$$
\mathrm{E}=\left(e_{i j}\right)_{n * n}=\left[\begin{array}{c}
1 \\
1-\frac{u_{21}-l_{21}}{2 m_{21}} \\
\vdots \\
1-\frac{u_{n 1}-l_{n 1}}{2 m_{n 1}}
\end{array}\right.
$$

$$
Q=M \times E
$$

In the formula, the matrix $\mathrm{M}$ is a matrix composed of all $m_{i j}$ in the fuzzy judgment matrix.

(4) Convert the adjusted judgment matrix Q into a judgment matrix $\mathrm{P}$ with a diagonal of 1 .

(5) Use analytic compatible matrix method to transform the judgment matrix $\mathrm{P}$ and derive the compatibility matrix $\mathrm{R}=\left(r_{i j}\right)_{n * n}$ :

$$
r_{i j}=\sqrt[n]{\prod_{k=1}^{n} P_{i k} \cdot P_{k j}}
$$

(6) Calculate the weight $w_{i}$ of this level:

$$
\begin{gathered}
w_{i}=\frac{c_{i}}{\sum_{k=1}^{n} c_{k}} \quad(\mathrm{i}=1,2, \ldots, \mathrm{n}) \\
c_{i}=\sqrt[n]{\prod_{k=1}^{n} r_{i k}} \quad(\mathrm{i}=1,2, \ldots, \mathrm{n})
\end{gathered}
$$

(7) Calculate the weight of each index relative to the general objective

Similarly, according to the above method, weight of other indexes below the criterion level is also calculated, and finally the weight of each index relative to the general objective is calculated. For example, the weight of criterion i relative to the general objective is $w_{i}$, and the

\subsection{Re-determine the index weight based on the TFN-AHP method}

The difference between triangular fuzzy number construction matrix and general analytic hierarchy process is that the former has a fuzzy judgment interval for the judgment of each index. Regarding meaning of this interval, there is uncertainty when experts compare the importance of elements in pairs, especially some criteria that require qualitative analysis, so to some extent, it indirectly reflects the uncertainty of the expert's judgment. It is obvious that greater interval range indicates greater uncertainty, and vice versa. Hence, based on the TFNAHP method, specific steps for re-determining the index weight are as follows:

(1) Construct a fuzzy judgment matrix

Experts make pairwise comparisons of the importance of indexes at the same level based on the upper-level criteria, and use triangular fuzzy numbers to construct a fuzzy judgment matrix. Suppose there are n elements, the constructed judgment matrix is $\mathrm{B}=\left(b_{i j}\right)_{n * n}$. Where, $b_{i j}=\left[l_{i j}, m_{i j}, u_{i j}\right]$ is a closed interval with $m_{i j}$ as the median, and $b_{j i}=b_{i j}{ }^{-1}=\left[\frac{1}{u_{i j}}, \frac{1}{m_{i j}}, \frac{1}{l_{i j}}\right]$

(2) Construct a fuzzy evaluation factor matrix $\mathrm{E}$ for the judgment matrix:

$\left.\begin{array}{ccc}1-\frac{u_{12}-l_{12}}{2 m_{12}} & \cdots & 1-\frac{u_{1 n}-l_{1 n}}{2 m_{1 n}} \\ 1 & \cdots & 1-\frac{u_{2 n}-l_{2 n}}{2 m_{2 n}} \\ \vdots & \ddots & \vdots \\ 1-\frac{u_{n 2}-l_{n 2}}{2 m_{n 2}} & \cdots & 1\end{array}\right]$

weight of $\mathrm{n}$ indexes under the influence of criterion $\mathrm{i}$ is $w_{j}(\mathrm{j}=1,2, \ldots, \mathrm{n})$, then the weight of these $\mathrm{n}$ indexes relative to the general objective is:

$$
\mathrm{w}=w_{i} \cdot w_{j}
$$

\section{TFN-AHP-based simulation of load shedding optimization algorithm}

\subsection{Power flow calculation based on wide area measurement system}

With the advantages of remote synchronous phasor measurement, high accuracy, rapid communication, and quick response, Wide Area Measurement System (WAMS) is suitable for large-scale power grids around the world. WAMS with PMU as the basic unit is a shared data platform that supports real-time data sharing across voltage levels, so load shedding after power flow transfer forms a system model across voltage levels. The wide area measurement system shares line power flow results under different voltage levels in real time. Based on this characteristic, this paper uses triangular fuzzy analytic hierarchy process to perform load shedding control on the calculated power flow. 


\subsection{Establish a fuzzy analytic hierarchy model}

The TFN-AHP algorithm has been introduced in the previous section, and now the simple system in Figure 1 is used for example simulation.

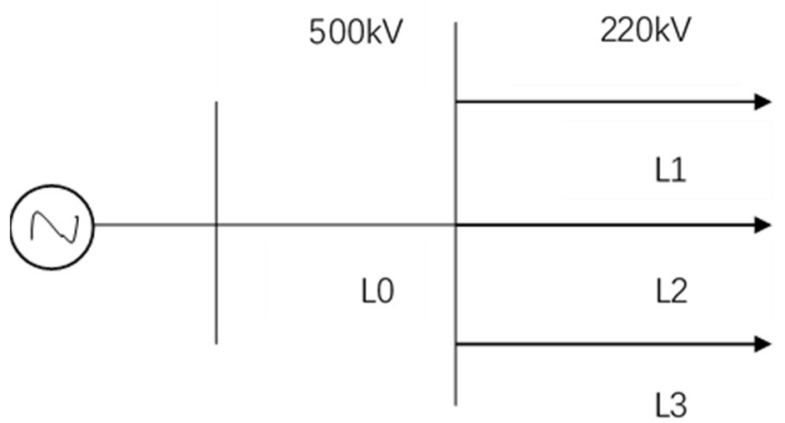

Figure 1 Simple power system

In Fuzzy Analytic Hierarchy Process, it is important to determine the evaluation index, so the factors influencing load shedding should be considered first. This paper selects three factors: line overload degree, load importance degree, and unit load shedding cost.

(1) Line overload degree is an important consideration factor, because line overload caused by power flow transfer is the direct cause of load shedding. If overload of the upstream line is caused by the overload of a downstream line, priority is given to shedding of serious overloaded lines. Moreover, line overload degree is a quantitative factor, and its calculation method can be expressed as:

Table 1 Basic situation of transmission lines

\begin{tabular}{|c|c|c|c|}
\hline Influencing factor & Line L1 & Line L2 & Line L3 \\
\hline Line overload degree & 1.0 & 0.8 & 0.6 \\
\hline $\begin{array}{c}\text { Load importance } \\
\text { degree }\end{array}$ & very important & $\begin{array}{c}\text { Relatively } \\
\text { important }\end{array}$ & important \\
\hline $\begin{array}{c}\text { Unit load shedding } \\
\text { cost }\end{array}$ & $882 \mathrm{RMB} / \mathrm{MW}$ & 998RMB/MW & $1195 \mathrm{RMB} / \mathrm{MW}$ \\
\hline
\end{tabular}

Using the previous method of building fuzzy judgment matrix, experts are invited to make pair-wise comparisons

and give rating to obtain the fuzzy judgment matrix of the three factors of the criterion layer, as shown in the following table 2 :

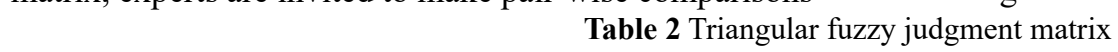

$$
\text { Line overload degree }=\frac{\text { real }- \text { time line load }}{\text { rated capacity }}
$$

Therefore, line overload degree can be quantified. The larger the value is, the higher the line overload degree is. It is very likely the reason for overload of the upstream line. Therefore, when the judgment matrix is formed, the line under overload has greater weight than other lines. response to the power system requirements, it is usually necessary to guarantee uninterrupted power supply for the first-level load, and try to ensure the uninterrupted power supply of second-level load, but when there is insufficient system power supply, power supply for third-level load can be stopped. Therefore, the transmission line that supplies power for the first-level load should have greater weight than that for the second-level load, while that supplying power for second-level load should have greater weight than that supplying power for third-level load.

(3) The unit load shedding cost is also an important factor. Load shedding should not only solve the upstream overload problem, but also consider economic efficiency, namely, the cost that the grid company needs to compensate the user after the load shedding. From the economic point of view, lines with lower unit load shedding costs should be removed first.

\subsection{Form a fuzzy judgment matrix}

First, given the first three factors, the statistical results of the three transmission lines under the $220 \mathrm{kV}$ voltage level were obtained as follows:
(2) Load importance degree is an important factor. In

\begin{tabular}{|c|c|c|c|}
\hline $\begin{array}{c}\text { Line overload } \\
\text { degree }\end{array}$ & Line overload degree & Load importance degree & Unit load shedding cost \\
\hline $\begin{array}{c}\text { Load } \\
\text { importance } \\
\text { degree }\end{array}$ & $(1.00,1.00,1.00)$ & $(0.50,0.80,1.00)$ & $(4.00,5.00,6.00)$ \\
\hline $\begin{array}{c}\text { Load } \\
\text { shedding cost }\end{array}$ & $(0.167,0.200,0.25)$ & $(1.00,1.00,1.00)$ & $(6.00,6.50,7.00)$ \\
\hline
\end{tabular}

The average consistency index of the matrix is calculated as $\mathrm{CR}=0$, which means that the matrix meets the consistency test requirement, and there is no need to re-adjust the matrix elements. 
According to formula (5), the weights of the three factors of line overload degree, load importance degree and unit load shedding cost are respectively: $\mathrm{W} 0=(0.4022$, $0.5178,0.0801$ ).

Among the three factors, load importance degree has relatively larger weight, followed by line overload degree, and unit load shedding cost has the smallest weight. In fact, it can be seen from the triangular fuzzy judgment matrix that load importance degree has a score greater than 1 compared with the other two, indicating that it is more important than the other two. Similarly, the importance of the load importance degree for line overload degree can also be calculated based on weight, which is 1.2874.

Then, determine the weight of the three lines under the three criteria:

(1) The weight of the three lines under overload

The data regarding overload degree of the three lines are shown in Table 3.

Table 3 Data of the three lines under overload

\begin{tabular}{|c|c|c|c|}
\hline & Line L1 & Line L2 & Line L3 \\
\hline $\begin{array}{c}\text { Line } \\
\text { L1 }\end{array}$ & $(1.00,1.00,1.00)$ & $(0.80,0.80,0.80)$ & $(0.60,0.60,0.60)$ \\
\hline $\begin{array}{c}\text { Line } \\
\text { L2 }\end{array}$ & $(1.25,1.25,1.25)$ & $(1.00,1.00,1.00)$ & $(1.00,1.00,1.00)$ \\
\hline $\begin{array}{c}\text { Line } \\
\text { L3 }\end{array}$ & $(1.67,1.67,1.67)$ & $(1.33,1.33,1.33)$ & $0.75,0.75)$ \\
\hline
\end{tabular}

The average consistency index of the matrix is calculated as $\mathrm{CR}=0$, which meets the consistency test requirement. Since line overload degree can be calculated quantitatively, according to Table 1, the importance of line $\mathrm{L} 1$ to line $\mathrm{L} 2$ is $0.8 / 1=0.80$. Hence, the fuzzy number is a certain value $(0.80,0.80,0.80)$. At the same time, according to the algorithm, the weight of the three lines under overload is calculated as W1 $=(0.2553,0.3191$, $0.4255)$.

(2) The weight of the three lines under load importance

The data of the three lines under load importance are shown in Table 4.

Table 4 Data of the three lines under load importance

\begin{tabular}{|c|c|c|c|}
\hline & Line L1 & Line L2 & Line L3 \\
\hline $\begin{array}{c}\text { Line } \\
\text { L1 }\end{array}$ & $(1.00,1.00,1.00)$ & $(2.00,2.50,3.00)$ & $(0.33,0.50,1.00)$ \\
\hline $\begin{array}{c}\text { Line } \\
\text { L2 }\end{array}$ & $(0.33,0.40,0.50)$ & $(1.00,1.00,1.00)$ & $(1.00,1.00,1.00)$ \\
\hline $\begin{array}{c}\text { Line } \\
\text { L3 }\end{array}$ & $(0.50,0.67,1.00)$ & $(1.00,2.00,3.00)$ & \\
\hline
\end{tabular}

The average consistency index of the matrix is calculated as $\mathrm{CR}=0.0032$, which meets the consistency test requirement. Since load importance can only be calculated qualitatively, according to Table 1, line L1 is more important than line L2, so the fuzzy number is an uncertain value $(2.00,2.50,3.00)$. Also, according to the algorithm, the weight of the three lines under overload is calculated as $\mathrm{W} 2=(0.4744,0.1800,0.3457)$.

(3) The weight of the three lines under the unit load shedding cost

The data of the three lines under the unit load shedding cost are shown in Table 5.

Table 5 Data of the three lines under unit load shedding cost

\begin{tabular}{|l|l|l|l|}
\hline & Line L1 & Line L2 & Line L3 \\
\hline Line L1 & $(1.00,1.00,1.00)$ & $(0.8837,0.8837,0.8837)$ & $(0.7381,0.7381,0.7381)$ \\
\hline Line L2 & $(1.1315,1.1315,1.1315)$ & $(1.00,1.00,1.00)$ & $(0.8351,0.8351,0.8351)$ \\
\hline Line L3 & $(1.3548,1.3548,1.3548)$ & $(1.1974,1.1974,1.1974)$ & $(1.00,1.00,1.00)$ \\
\hline
\end{tabular}

The average consistency index of the matrix is calculated as $\mathrm{CR}=0$, which meets the consistency test requirement. Since the unit load shedding cost can be calculated quantitatively, according to Table 1, the importance of line L1 to line L2 is $882 / 998=0.8837$, so the fuzzy number is a certain value $(0.8837,0.8837,0.8837)$. A lower unit load shedding cost means it is more likely to be preferentially removed. At the same time, according to the algorithm, the weight of the three lines under the unit load shedding cost is calculated as W3 $=(0.2868,0.3246$, 0.3886).

Finally, the comprehensive weight of each program against the general objective is calculated by:

$\mathrm{W}=\mathrm{W} 0 *[\mathrm{~W} 1, \mathrm{~W} 2, \mathrm{~W} 3]^{\mathrm{T}}=(0.3713,0.2475,0.3813)$
According to the comprehensive weight, line L2 has the lowest weight, followed by line L1, and then line L3. Therefore, priority should be given to line L2 when selecting load shedding lines.

\section{Conclusion}

The $500 \mathrm{kV}$ power grid power flow transfer overload will cause malfunction in relay protection, so it is necessary to quickly eliminate the overload phenomenon. This paper comprehensively utilized the wide area measurement system to share in real time the power flow analysis of the lines under different voltage levels, and proposed a cross- 
voltage level load shedding optimization strategy based on the fuzzy analytic hierarchy process. It mainly analyzed the influence of the three factors of line overload degree, load importance degree and unit load shedding cost, and used a simple system for example simulation, which scientifically found the line that needed to be cut from the low-voltage level lines. The strategy solves the overload problem of the upstream line and minimizes the impact on users, which has great practicability for practical engineering applications.

\section{References}

1. Li Xiuqing, Cai Zexiang. Low-frequency load shedding control optimization algorithm of power system[J]. Automation of Electric Power Systems, 1998(10): 23-25.

2. Jiang Zhen. Research on power flow transfer based on wide area information [D]. Huazhong University of Science and Technology, 2010.

3. Xu Huiming, Bi Tianshu, Huang Shaofeng, et al. Control strategy for preventing chain trip based on wide area synchronous measurement system[J]. Proceedings of the Chinese Society for Electrical Engineering, 2007, 27(19): 32-38.

4. Li Xiang, Zhang Guoqing, Guo Zhizhong. A joint load shedding scheme based on the transmission section N-1 static secure power flow constraint [J]. Automation of Electric Power Systems, 2004, 28(22): 42-44.

5. Luo Baofeng, Wang Qingxian, Zhu Junhu, et al. A method for determining the weights of evaluation indexes based on triangular fuzzy numbers and analytic hierarchy process $[\mathrm{J}]$. Research on Telecommunication Technology, 2013(6): 9-16. 